\title{
Food Insecurity and Cardiovascular Disease Risk
}

\author{
Yibin Liu ${ }^{1}$ (1) $\cdot$ Heather A. Eicher-Miller ${ }^{2}$ \\ Accepted: 9 March 2021 / Published online: 27 March 2021 \\ (C) The Author(s), under exclusive licence to Springer Science+Business Media, LLC, part of Springer Nature 2021
}

\begin{abstract}
Purpose of Review This review examines the current epidemiological evidence for the relationship between levels of food insecurity and cardiovascular disease (CVD) outcomes among US adults $>17$ years.

Recent Findings Review of recent literature revealed that reduced food security was associated with decreased likelihood of good self-reported cardiovascular health and higher odds of reporting CVD-related outcomes such as coronary heart disease, angina, heart attack, peripheral arterial disease, and hypertension.

Summary Existing evidence suggests a compelling association between each level of reduced food security and CVD risk with a particularly strong association between very low food security and CVD risk. Policies and public health-based strategies are needed to identify the most vulnerable subgroups, strengthen and enhance access to food assistance programs, and promote awareness and access to healthful foods and beverages to improve food security, nutrition, and cardiovascular health.
\end{abstract}

Keywords Food security $\cdot$ Cardiovascular disease $\cdot$ Heart disease $\cdot$ Health outcomes $\cdot$ Nutrition $\cdot$ Risk factor

\section{Introduction}

Cardiovascular disease (CVD) is the number one leading cause of death globally [1•]. In the USA, CVD death was primarily attributable to coronary heart disease (CHD, $42.6 \%)$, followed by stroke (17.0\%), hypertension (10.5\%), heart failure $(9.4 \%)$, and diseases of arteries $(2.9 \%)$; other minor CVD causes combined accounted for $17.6 \%$ in 2017 [2]. CVD poses a substantial health and economic burden in the USA. About $10.6 \%$ of US adults were living with CHD, heart failure, or stroke between 2013 and 2016 [3]. The estimated annual direct and indirect cost of CVD was $\$ 351.2$ billion for 2014-2015, and the total cost is expected to reach $\$ 1.1$ trillion in $2035[1 \bullet]$. Although estimates suggest that

This article is part of the Topical Collection on Nutrition

Yibin Liu

yibinliu@buffalo.edu

Heather A. Eicher-Miller

heicherm@purdue.edu

1 Department of Community Health and Health Behavior, School of Public Health and Health Professions, University at Buffalo, 314

Kimball Tower, Buffalo, NY 14214, USA

2 Department of Nutrition Science, Purdue University, West Lafayette, IN, USA approximately $80 \%$ of CVD can be prevented by controlling risk factors and adopting a healthy lifestyle, currently in the USA, the prevalence of heart healthy behaviors is low, and substantial barriers to implementation exist including low socioeconomic status and food insecurity $[1 \bullet, 4,5]$.

Food insecurity is a major US public health concern, with $10.5 \%$ of households having limited access at all times to enough food for an active and healthy life in 2019 [6]. Due to the coronavirus disease 2019 (COVID-19) pandemic, food insecurity is estimated to have tripled to $38.3 \%$ and increased well beyond levels seen during the Great Recession, based on a representative national sample of US adults collected in March 2020 [7]. The prevalence of food insecurity was even higher (44\%) for US adults with income below $250 \%$ of the federal poverty level [8]. Growing evidence reveals food insecurity to be a risk factor for poor health across the life course. Children and adults living in food insecure households may have increased risk for negative health outcomes such as poor development, overall poor health, adverse mental health conditions, chronic diseases, functional limits, and potentially shorter life expectancy compared with those living in food secure households [9-13]. To cope with food insecurity, households may rely on a low variety of nutritionally inadequate foods and have disordered eating patterns that contribute to poor overall dietary quality, and do not meet the recommendation for key food groups nor fulfill the average requirement for nutrients $[5,11,14-19]$. Indeed, the classification of 
food security defines ranges; in the most severe level, very low food security (VLFS), amount of food is reduced, and disrupted eating patterns are present [20]. Low food security (LFS) is comparatively less severe but defines a range where there is reduced quality or variety but not amount of food [20]. In marginal food security (MFS), anxiety of having enough food exists, but no changes in diet are present, and finally, in high food security (HFS), there are no indications of food access limits [20]. Compromised dietary intake present with LFS and VLFS combined with potential cycles of bingeing and fasting alongside the fluctuating nature of food insecurity may lead to adverse metabolic effects such as insulin resistance and poor weight management that heighten CVD risk $[1 \cdot 11,21,22]$. Additionally, lower food security may affect CVD risk by activating the stress response and elevating depressive and anxiety symptoms that could potentially trigger unhealthful coping behaviors or limit one's ability to manage disease [21, 22].

Dietary intake and other lifestyle factors associated with and independent from food insecurity are important to CVD risk. Research using comparable risk assessment methods and nationally representative data have shown that about $45 \%$ of cardiometabolic deaths were attributable to poor dietary habits [23]. The leading dietary risk factors for cardiometabolic deaths included low consumption of vegetables and fruits $(15 \%)$, high sodium intake $(10 \%)$, low consumption of nuts/seeds (9\%), high consumption of processed meats (8\%), low intake of seafood omega-3 fats (8\%), and high consumption of sugar-sweetened beverages (7\%) [23], many of which have been associated with food insecurity [5, 15, 24, 25]. Besides eating a healthy diet, the American Heart Association's Life's Simple 7 highlights three other health behaviors (non-smoking/non-smokeless tobacco use, physical activity, healthy body weight) and three CVD risk factors (blood glucose, total cholesterol, and blood pressure) important for heart health $\left[1^{\bullet}\right]$. Poor adherence to these modifiable risk factors has been associated with reduced food security, indicating the great potential of food insecurity to be associated with cardiovascular health $[9,11,24,26]$. Therefore, a strong link between food insecurity and CVD risk is plausible. Several studies have evaluated this relationship, but there is a lack of synthesis of recent literature to determine how the severity or the range of food insecurity may be associated with CVD risk. Summarizing the comprehensive evidence for this relationship may inform specific intervention strategies targeting food insecure groups by food security level to reduce CVD risk. This review examines the current epidemiological evidence for the relationship between the range of food insecurity and CVD outcomes among US adults $>17$ years of age and concentrates on papers that reflect the most recent work. An explanation of the proposed mechanisms and policy implications is also included in discussion of the findings.

\section{Methods}

A PubMed search of the terms "food security" and "health outcomes" or "chronic disease" or "cardiovascular disease" or "heart disease" or "coronary heart disease" or "angina pectoris" or "myocardial infarction" or "heart attack" or "peripheral arterial disease" or "stroke" or "cerebrovascular disease" or "heart failure" or "arrhythmia" or "rheumatic heart disease" or "congenital heart disease" or "thrombosis" or "pulmonary embolism" yielded 262 peer-reviewed studies published between 2010 and 2020. Searches were limited to human studies published in the English language. First, the title and abstract were assessed for general relevance. Second, the full text of remaining articles was examined for accordance with eligibility criteria. Relevant articles were included in this review if they (1) used a sample drawn from the US population, (2) contained self-reported or measured CVDrelated outcomes, and (3) classified food insecurity using the US Department of Agriculture (USDA) Household Food Security Survey Module or variants on this measure.

\section{Results}

After an examination of the title and abstract, 234 articles were excluded from further consideration (Fig. 1). Full texts of the remaining 28 articles were assessed in accordance with the eligibility criteria, and 12 articles were retained for inclusion. Two additional eligible articles were identified through review of article references. A total of 14 studies [27-29, 30••, 31-40] met all criteria and were included in the final review (Table 1).

Most of the research examining food insecurity and its association with CVD concentrated on working-age adults or adults in general (Table 1), except for three studies that featured older adults [29, 33, 37]. Eleven studies used a nationally representative sample $[27,29,30 \bullet \cdot, 32,33,35-40]$, while three studies used convenience samples $[28,31]$ or state-specific representative samples [34]. Twelve studies were cross-sectional $[28,29,30 \bullet \cdot, 31-39]$, and one study was ecological [40], whereas one used longitudinal analysis [27]. Four studies focused on evaluating the relationship between food insecurity and CVD outcomes among low-income populations that fell below a certain percentage of the federal poverty level $[27,30 \bullet \cdot, 31,36]$. High prevalence of heart disease $(18 \%)$ and stroke (12\%) above the national average [41] were reported specifically in a food pantry user population (aged 21-80 years) where the majority lived below $100 \%$ federal poverty level [31]. Only one study used clinical measures to ascertain peripheral arterial disease [33], while the majority used self-reported data for CVD-related outcomes [27, 30••, 31, 32, 34-38]. Additionally, three studies calculated the 10-year risk for atherosclerotic CVD events [28, 29, 39] using the pooled cohort equations introduced by the American 


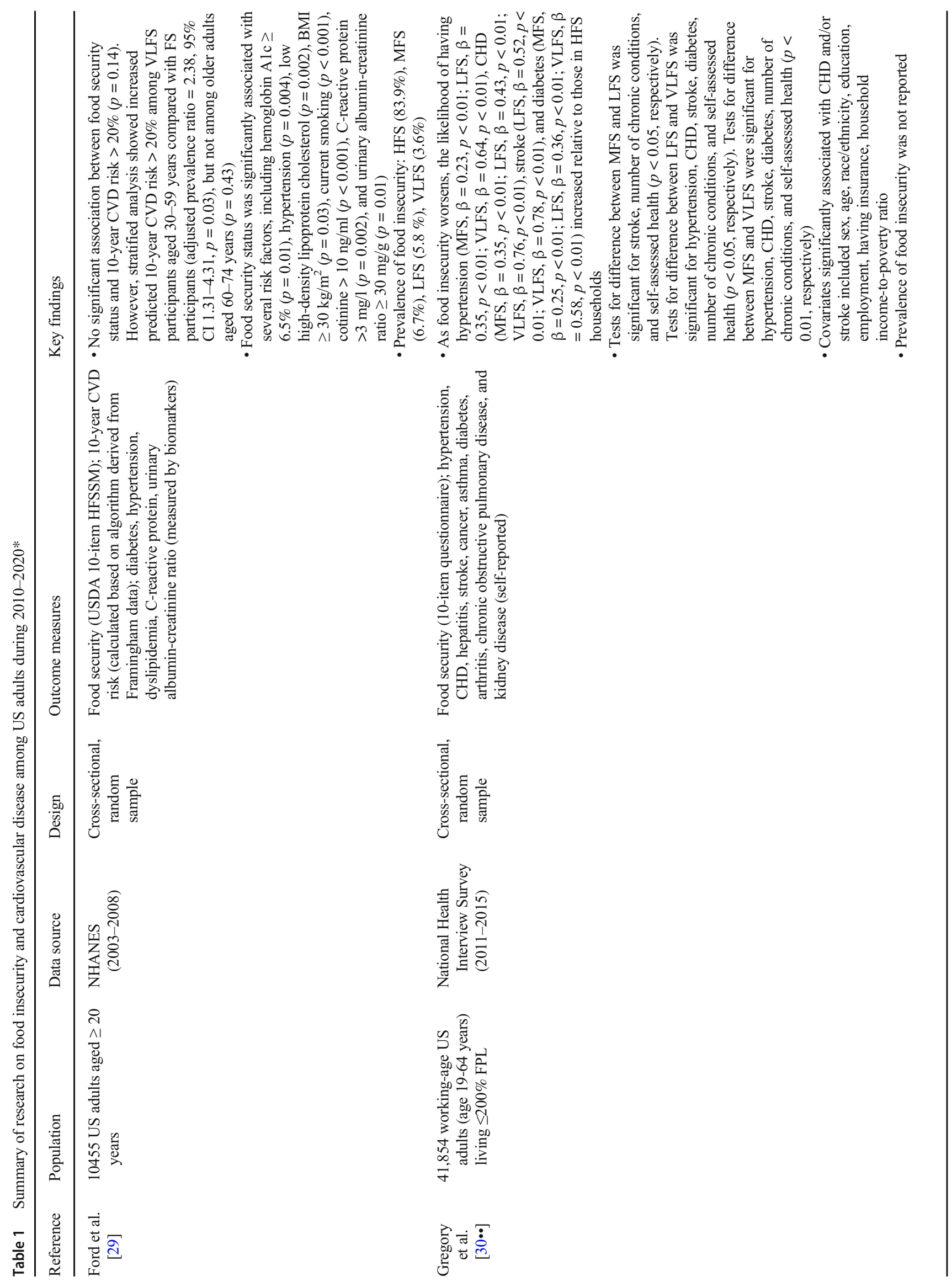




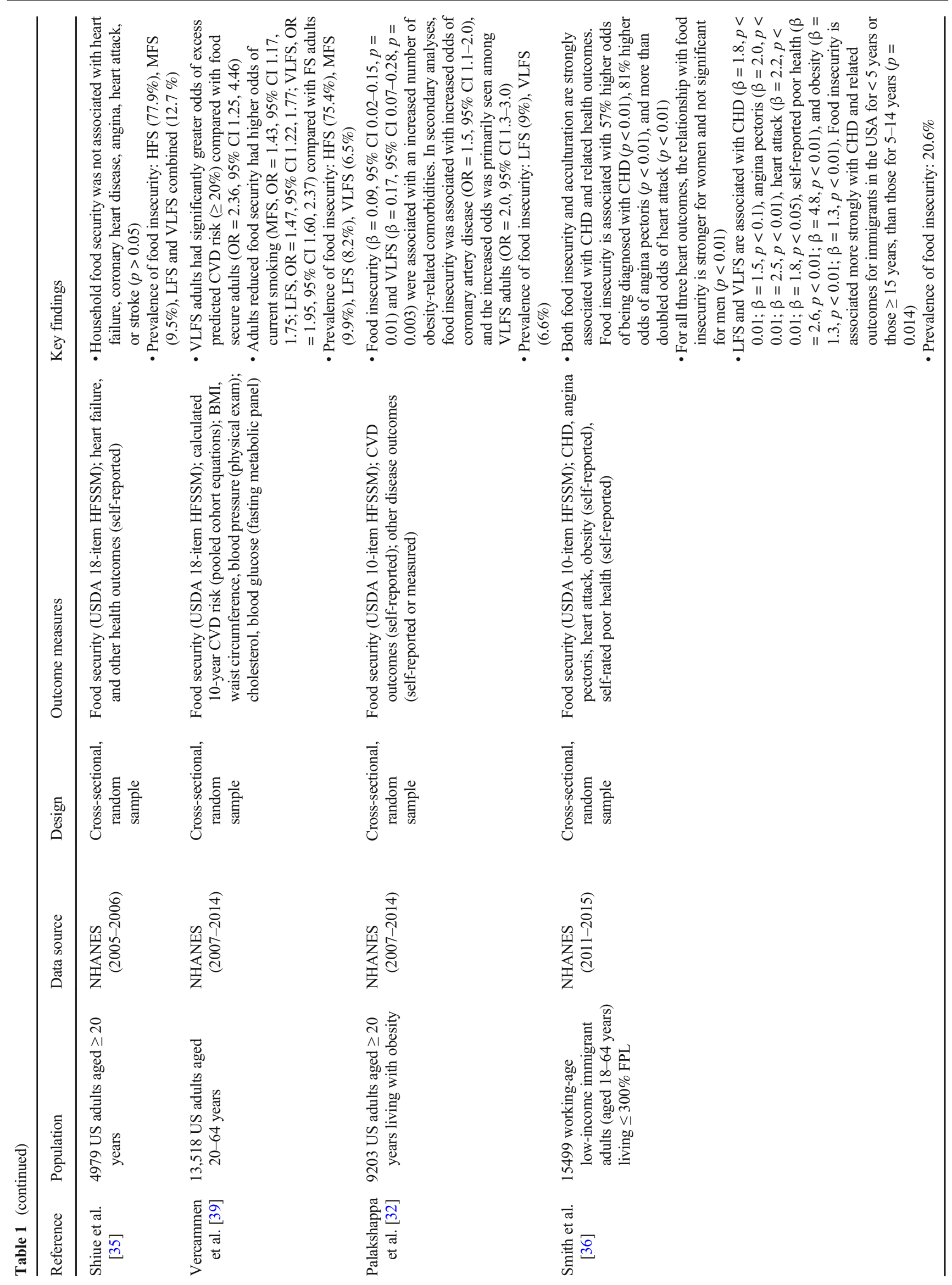




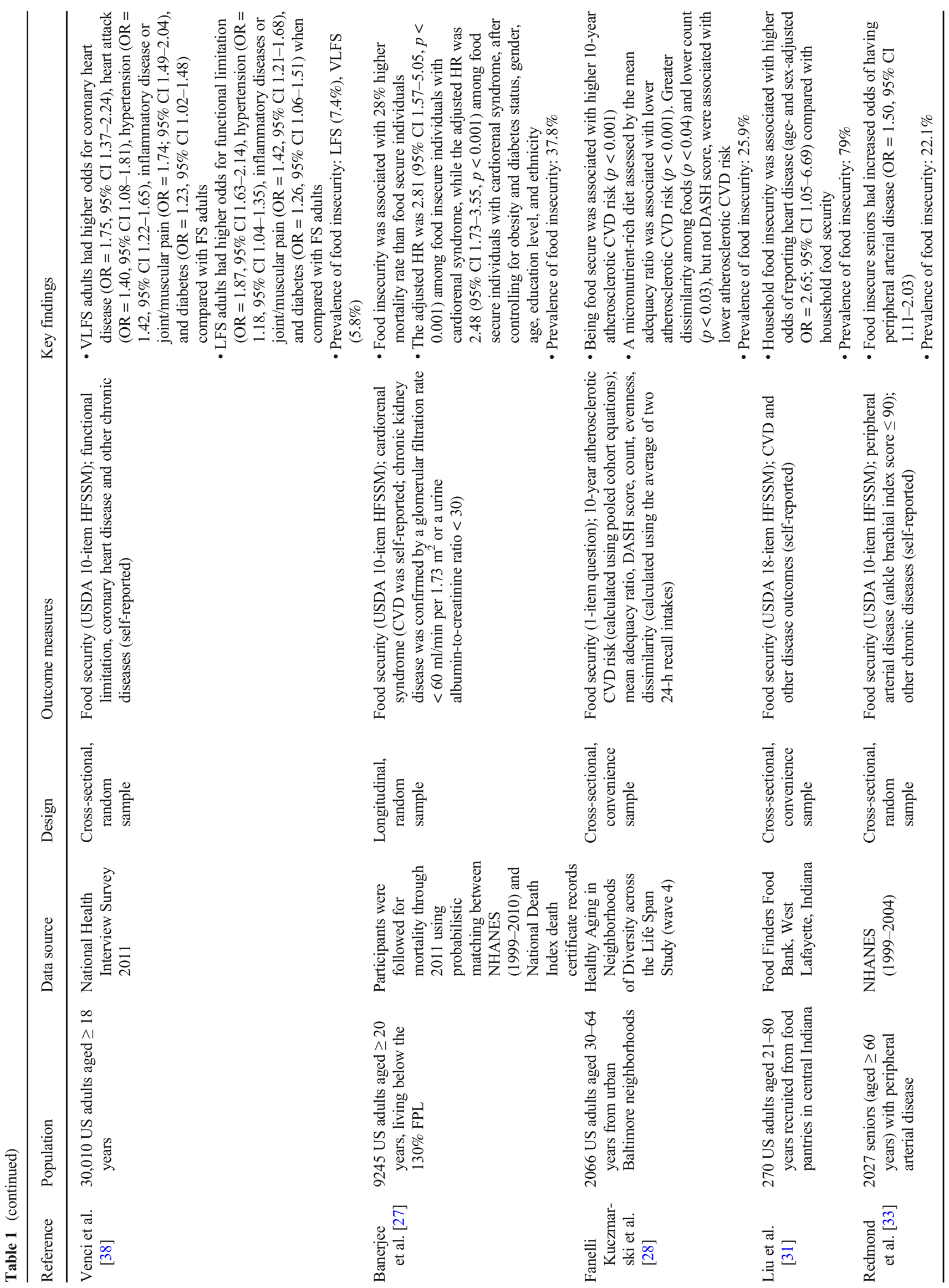




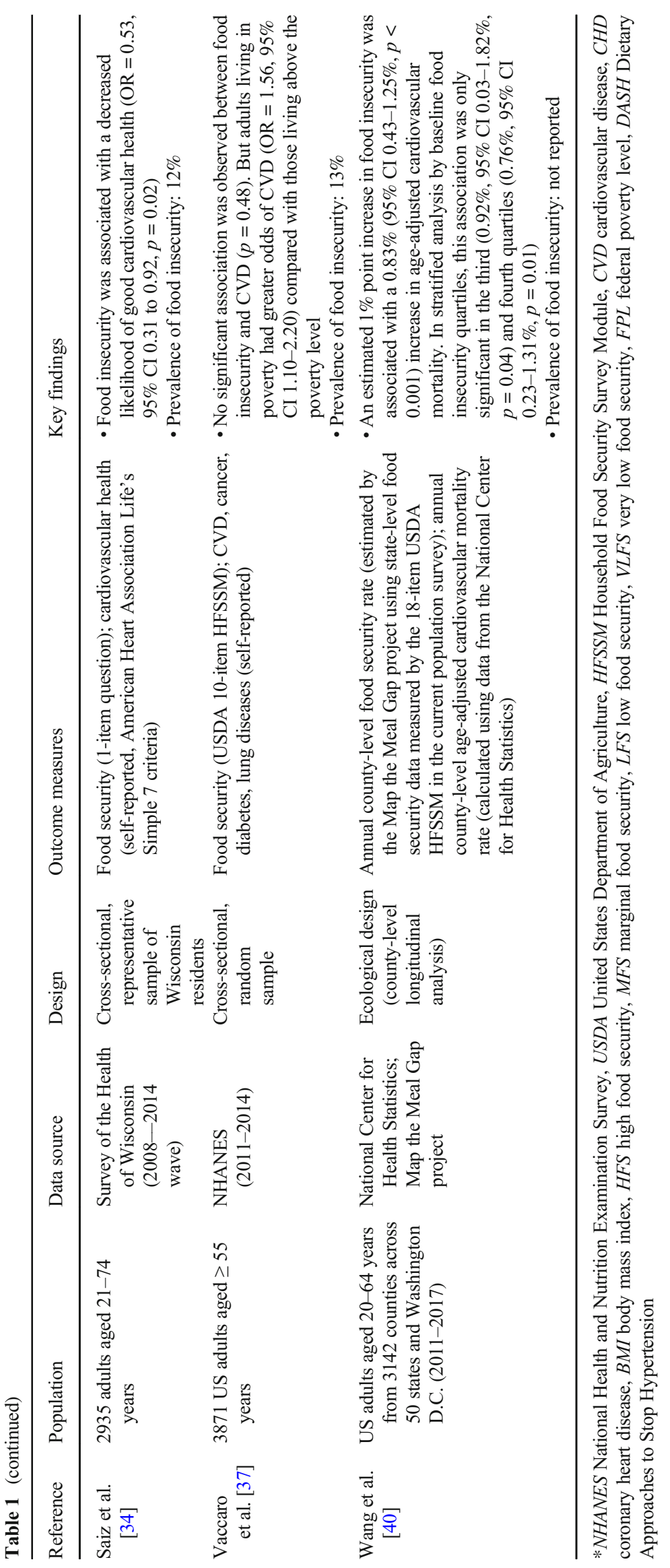


Fig. 1 Flowchart of the literature search and article selection process

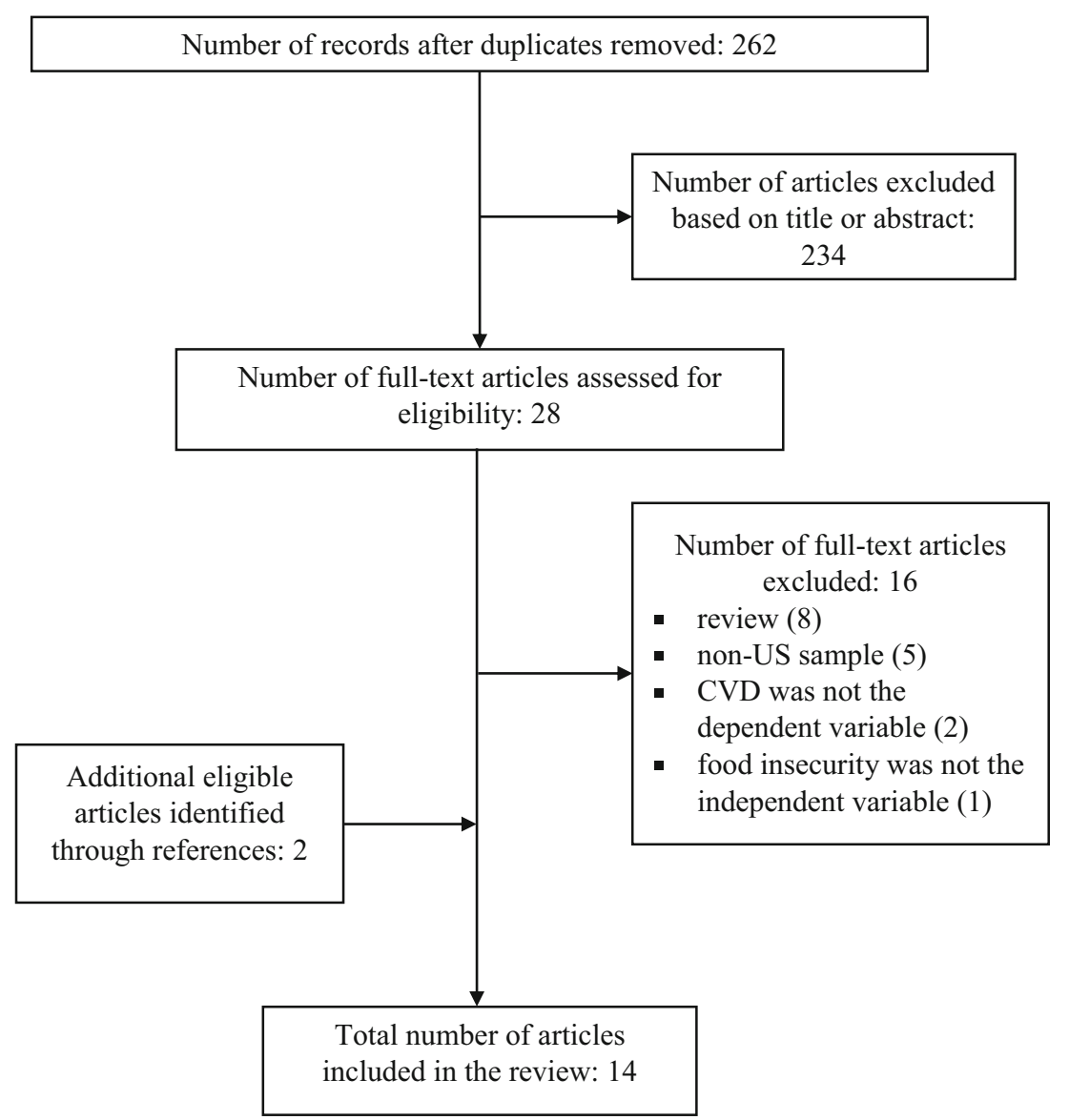

College of Cardiology/American Heart Association [42], based on risk factors such as age, total cholesterol, blood pressure, high-density lipoprotein cholesterol, type 1 or 2 diabetes status, and smoking status. Food security status was quantified by the 18-item or 10-item USDA's Household Food Security Survey Module in 12 studies [27, 29, 30••, 31-33, 35-40], while two studies used a 1-item or 2-item screener to assess food security $[28,34]$. The prevalence of food insecurity ranged from $9.4 \%$ [29] to $79 \%$ [31].

Four studies in this review classified food security into HFS, MFS, LFS, and VLFS [29, 30••, 35, 39], while three studies combined HFS and MFS as being food secure (FS) that was then examined along with LFS and VLFS [32, 36, 38]. These studies suggested a compelling association between each level of reduced food security and CVD risk, though evidence supporting a dose-dependent relationship was mixed. According to the USDA report conducted using data from the National Health Interview Survey of over 40,000 working-age adults living $\leq 200 \%$ federal poverty level, adults in households with MFS, LFS, and VLFS all had significantly increased risk of CHD, hypertension, diabetes, and four other chronic illnesses (asthma, arthritis, chronic obstructive pulmonary disease, kidney disease), compared to those in HFS households [30••]. As food insecurity worsened, the risk of chronic illness was progressively higher as suggested by the statistically significant differences between MFS, LFS, and VLFS groups [30••]. Findings from another study did not support a consistent dose-dependent relationship between food insecurity and CVD-related outcomes. Specifically, MFS, LFS, and VLFS were associated with higher odds of CHD $(\beta=1.5 \pm 0.3, p<0.1 ; \beta=1.8 \pm 0.3$, $p<0.01 ; \beta=1.5 \pm 0.3, p<0.1)$, angina pectoris $(\beta=2.2 \pm 0.5$, $p<0.01 ; \beta=2.0 \pm 0.4, p<0.01 ; \beta=2.5 \pm 0.6, p<0.01)$, and heart attack $(\beta=0.7 \pm 0.2, p>0.1 ; \beta=2.2 \pm 0.6, p<0.01 ; \beta=$ $1.8 \pm 0.5, p<0.05)$ compared with HFS among low-income adults living $\leq 300 \%$ federal poverty level $(n=15,499)$ from the National Health and Nutrition Examination Survey (NHANES) [36]. Similar findings were reported in another study using NHANES data where only VLFS adults had significantly higher odds for CHD and heart attack and both LFS and VLFS adults had significantly higher odds for hypertension, diabetes, and inflammatory disease or joint/muscular pain, compared with FS adults [38]. Another study pointed out that the significantly greater odds of CHD $(\mathrm{OR}=1.5$, 95\% CI 1.1-2.0) observed among food insecure adults was primarily seen among individuals living in VLFS households $(\mathrm{OR}=2.0,95 \%$ CI 1.3-3.0), compared to their FS counterparts based on NHANES data $(n=9,203)$ [32]. Using data from NHANES of over 13,000 adults aged 20-64 years, one study showed 2.4 times greater odds of excess predicted 10- 
year CVD risk ( $\geq 20 \%$ ) among VLFS adults compared with FS adults [39]. Another study showed significantly increased predicted 10-year CVD risk (> $20 \%$ ) among VLFS adults aged 30-59 years compared with FS adults (adjusted prevalence ratio $=2.38, p=0.03$ ), but not among older adults aged 60-74 years [29]. Of these seven studies, only one reported no significant association between food insecurity and heart failure, CHD, angina, heart attack, or stroke [35].

The other seven studies used binary comparisons of adults living in food insecure households with those living in FS households [27, 28, 31, 33, 34, 37, 40]. Overall, food insecurity was associated with significantly decreased likelihood of good self-reported cardiovascular health $(\mathrm{OR}=0.53,95 \% \mathrm{CI}$ 0.31-0.92) [34], higher odds of reporting CVD-related outcomes such as peripheral arterial disease $(\mathrm{OR}=1.50,95 \% \mathrm{CI}$ 1.11-2.03) [33] and heart disease ( $\mathrm{OR}=2.65,95 \%$ CI 1.05 6.69) [31], and higher mortality rate among individuals with cardiorenal syndrome (HR $=2.81,95 \%$ CI 1.57-5.05) [27]. The ecological study using county-level longitudinal analysis of data from 3142 US counties across 50 states and Washington DC (2011-2017) showed that a $1 \%$ point increase in food insecurity was associated with a $0.83 \%(95 \%$ CI $0.43-1.25 \%, p<0.001)$ increase in age-adjusted cardiovascular mortality [40]. Surprisingly, one study using data from a convenience sample of 2066 US adults aged 30-64 years showed that living in FS households was associated with higher 10-year CVD risk $(p<0.001)$ relative to food insecure households, though the study used a single item question to assess food security status and thus may be subjected to misclassification bias [28]. Another study using NHANES data reported no association between food insecurity and selfreported CVD outcome among older adults aged $\geq 55$ years; however, the study reported that adults living in poverty had greater odds of CVD (OR $=1.56,95 \%$ CI 1.10-2.20) compared with those living above the poverty level [37].

\section{Discussion}

This review of the recent literature suggests a compelling association between reduced food security and CVD risk. The association between VLFS with CVD-related outcomes was particularly strong; however, each level of food insecurity and even MFS presented a unique risk for CVD. The full range of food insecurity classification to four levels captures important information about economic hardship translated to poor health outcomes, showing statistically significant differences between each level across the range of food insecurity in terms of likelihood of various chronic health outcomes [30••]. For most chronic health outcomes examined in the USDA report, MFS, LFS, and VLFS were strongly associated with increased risk and increased number of chronic conditions, relative to HFS $[30 \bullet \cdot$. Specifically, there was a statistically significant increase in the prevalence of hypertension for adults as food security worsens (HFS, 20\%; MFS, 24\%; LFS, 28\%; VLFS, $36 \%$ ) [30••]. Another study supported the notion that MFS was associated with higher cumulative biological risk scores and MFS adults were more likely to have higher mean glycohemoglobin, C-reactive protein, and systolic blood pressure, relative to HFS [43]. Yet the relationship of MFS to CVD may not be observed when food security status is dichotomized [10]. LFS and VLFS were both associated with CVD-related outcomes [27, 29, 30••, 31-34, 36, 38-40]; in particular, VLFS adults had at least two times higher odds of having excess 10 -year CVD risk $(\geq 20 \%)$ and at least 1.4 times higher odds of having CHD, heart attack, and hypertension compared to FS adults $[29,30 \bullet \cdot, 32,38,39]$. One study reported that VLFS adults had the highest likelihood of having CHD, hypertension, and stroke across the four levels of food insecurity $[30 \bullet \bullet$.

The mechanisms by which reduced food security may cause or heighten CVD risk remain unclear, but previous studies hypothesized several pathways by which reduced food security could lead to the development or worsening of chronic disease and health conditions. In addition to the mechanisms described in the introduction of this paper, previous life experiences of food insecurity may heighten CVD risk, yet these remain uninvestigated hypotheses. For example, the experience of food deprivation as a child may have lifelong influence on eating patterns, attitudes, and preference in adulthood; some adults who experienced food shortages in childhood described longterm disordered eating practice, such as bingeing when food was available or food hoarding $[44,45]$. Food insecurity has also been associated with increased depressive symptoms and stress [13]. Stress activates the sympathetic-adrenal-medullary system and the hypothalamic-pituitary-adrenal axis, which trigger the fight-or-light responses and a surge in stress-mediator hormones that act on multiple biological systems [46]. Thus, stress has the potential to affect blood glucose level, serum lipids, and blood pressure, which have implications on CVD outcomes [46]. Chronic stress may lead to increased allostatic load, a concept referring to the excessive wear and tear on multiple physiological systems due to the chronic overuse and imbalance of the stress mediators [47, 48]. Based on the allostatic load framework, one study created a cumulative biological risk score using data from NHANES and found that food insecurity was associated with a 0.14-unit higher cumulative biological risk score $(p=0.003)$ and 1.2 times higher odds of elevated biological risk ( $p=0.003$ ), which indicated a potential role of food insecurity in influencing chronic health outcomes through chronic stress [43]. In addition, stress may further reduce cognitive bandwidth, motivation, and self-efficacy, which may undermine efforts at self-management or trigger unhealthful behaviors such as smoking [13, 14].

Reduced ability to manage complex chronic conditions may increase the risk of developing or worsening CVD among 
food insecure populations. Some researchers suggested a bidirectional relationship between food insecurity and cardiovascular health [39, 49]. Food insecure individuals were reported to have higher odds of delaying medication, postponing needed medical care, and hospitalization compared with food secure individuals [50, 51]. Cost-related medication underuse is a term used to describe reducing, skipping, or delaying use of medications or using lower-cost medications to compensate for lack of resources [52]. A significant doseresponse relationship between cost-related medication underuse and food insecurity was reported among older adults, with increasing likelihood of cost-related medication underuse with increasing severity of food insecurity [52]. Studies have also associated food insecurity with impaired glucose self-monitoring and poor metabolic control among diabetic patients [9, 11, 49]. Conversely, it is also possible that worsening cardiovascular health may increase medical expenses and reduce capacity for maintaining employment, leading to lower income and increasing risk of food insecurity $[39,49]$. One study suggested that individuals living with food insecurity incurred an additional \$1863 annually in healthcare expenditure, $\$ 493$ more for inpatient hospitalizations, and $\$ 779$ more for prescription medications [49]. The estimated annual total healthcare expenditures were higher among food insecure individuals with heart disease $(\$ 5144.1, p<0.0001)$, hypertension $(+\$ 2175.5, p=0.003)$, and diabetes $(+\$ 4414.6$, $p=0.004)$, compared with FS individuals with these conditions [49]. Another study also showed that food insecurity was associated with higher use of emergency visits, inpatient admissions, and having high healthcare costs [53].

Most studies included in this review were cross-sectional and focused on working-age adults or adults in general, while the senior population received little attention. According to a Feeding America report, food insecure seniors were 19\% more likely to have hypertension, $57 \%$ more likely to have congestive heart failure, $66 \%$ more likely to have experienced a heart attack, $65 \%$ more likely to be diabetic, twice as likely to report fair or poor general health, 2.3 times more likely to suffer from depression, and over $30 \%$ more likely to report at least one limitation in activities of daily living compared with food secure seniors [26]. A subgroup of concern is food insecure seniors with income below $200 \%$ of the federal poverty level. Food insecurity rate was highest among seniors with incomes below the poverty line $(30 \%)$, compared to those with incomes between 100 and $200 \%$ of the federal poverty level $(18 \%)$ or those with income above $200 \%$ of the federal poverty level (4\%) [26]. Food insecure seniors with income below $200 \%$ of the federal poverty level were more likely to have $\mathrm{CHD}$, congestive heart failure, heart attack, chest pain, hypertension, high cholesterol, diabetes, depression, and other negative health outcomes compared with their food secure counterparts [26]. Additionally, seniors living with limitation in activities of daily living experience difficulty in performing daily functions such as going to the grocery store to purchase food, eating, or bathing [26]. Future research is warranted to assess how levels of food insecurity affect CVD-related outcomes among older adults, especially those living with functional limits and below $200 \%$ of the federal poverty level.

Food insecurity and its association with CVD present challenges to policy-makers, program administrators, and healthcare providers in the USA. With the COVID-19 pandemic causing a never-before-seen food insecurity crisis and economic downturn in the USA, existing disparities related to food insecurity are exacerbated and may continue to exert acute and long-lasting health consequences disproportionately felt by low-income populations [54, 55]. The summarized evidence supports the need for strengthening existing successful strategies such as federal nutrition assistance, nutrition education, and implementation of potentially new strategies to improve food security. For example, the Supplemental Nutrition Assistance Program (SNAP) has been established since 1964 and shown to successfully improve household food security [56-58]. Expansion of the SNAP program may contribute to further reduction in food insecurity, which in turn may lead to the potential reduction in CVD burden [59, 60]. Many people who are eligible for SNAP do not participate in this entitlement program as suggested by a national average participation rate of $84 \%$ in 2017 [61]. Especially among seniors, over $60 \%$ of those eligible do not receive benefits, suggesting room for improvement in reach of the current SNAP program [9]. Nutrition education has also been shown to improve food security $[62,63]$. Education through the Supplemental Nutrition Assistance Program-Education (SNAP-Ed) combines nutrition and budgeting education for those who qualify for SNAP; SNAP-Ed improved food security by $25 \%$ over a 1 -year timeframe in an intervention compared with a control group that was independent of participation in SNAP or other food assistance $[62,63]$. Yet SNAP-Ed is even more restricted as a non-entitlement program serving approximately 5\% of US adults who used SNAP in 2018 [64, 65]. With food insecurity incurring an additional $\$ 77.5$ billion in healthcare expenditure annually, the "return on investment" potential of expanding food insecurity reduction programs like SNAP and SNAP-Ed may be significant [49].

Understanding the extent to which food insecure populations achieve dietary recommendations is the foundation for tailoring powerful public health-based strategies to "meet people where they are" to help them make small and positive shifts [66]. Interventions that integrate food insecurity screening into routine care (at clinics or dietitian's office) may be an important step to identify individuals experiencing food insecurity and subsequently improve chronic health conditions and patient care for vulnerable populations [67-70]. Emergency food pantries have been increasingly used as a dependable food source over the last decade and chronic use may continue to increase with guidelines that further restrict 
SNAP eligibility $[71,72]$. Programs that link patients to emergency food pantries and other community resources to help support unmet need for food assistance may also be helpful [73]. Lastly, supporting access to healthful foods and beverages in food environments such as federal food assistance programs and emergency food assistance network may be particularly relevant for disadvantaged subgroups to improve diet, health, and wellness [74].

\section{Conclusions}

This review suggests a compelling association between reduced food security and CVD risk with a particularly strong link between VLFS with CVD risk and evidence that each range of food security presents a unique risk for CVD. Future research using longitudinal individual-level data focusing on CVD outcomes will likely allow researchers to examine how different factors mediate the observed relationships between reduced food security and CVD risk over time, with appropriate adjustment of covariates such as cigarette smoking and physical activity. Policies and public healthbased interventions are needed to identify the most vulnerable subgroups experiencing food insecurity, strengthen and enhance access to food assistance programs, and promote awareness and access to healthful foods and beverages to improve nutrition, food security, and cardiovascular health.

\section{Compliance with Ethical Standards}

Conflict of Interest Yibin Liu declares no conflict of interest. Heather A. Eicher-Miller reports grants from the United States Department of Agriculture, National Institute of Food and Agriculture, Hatch Project 1019736; personal fees from Mead Johnson, National Dairy Council, and American Egg Board; and grants from Eli Lilly and Company.

Human and Animal Rights and Informed Consent This article does not contain any studies with human or animal subjects performed by any of the authors.

\section{References}

Papers of particular interest, published recently, have been highlighted as:

- Of importance

•- Of major importance

1. Virani SS, Alonso A, Benjamin EJ, et al. Heart Disease and Stroke Statistics-2020 update: a report from the American Heart Association. Circulation. 2020;141(9):e139-596. This report provides the most up-to-date statistics regarding cardiovascular diseases and risk factors that contribute to cardiovascular health and related outcomes.
2. Blackwell DL, Villarroel MA. Tables of summary health statistics for US adults: 2017 National Health Interview Survey. Atlanta: Centers for Disease Control and Prevention. http://www.cdc.gov/ nchs/nhis/SHS/tables.htm. Accessed 16 Oct 2020; 2019.

3. National Center for Health Statistics. National Health and Nutrition Examination Survey (NHANES) public use data files. Atlanta: Centers for Disease Control and Prevention. https://www.cdc.gov/ nchs/nhanes/. Accessed 12 Nov 2020; 2020.

4. Yang Q, Cogswell ME, Flanders WD, et al. Trends in cardiovascular health metrics and associations with all-cause and CVD mortality among US adults. JAMA. 2012;307(12):1273-83.

5. Hanson KL, Connor LM. Food insecurity and dietary quality in US adults and children: a systematic review. Am J Clin Nutr. 2014;100(2):684-92.

6. Coleman-Jensen A, Rabbitt MP, Gregory CA, Singh A. Household food security in the US in 2019. Alexandria: US Department of Agriculture Economic Research Service. https://www.ers.usda. gov/publications/pub-details/?pubid=99281. Accessed 16 Oct 2020; 2020.

7. Fitzpatrick K, Harris C, Drawve G. Assessing US food insecurity in the United States during COVID-19 pandemic. Fayetteville: AR Community and Family Institute, University of Arkansas. https:// fulbright.uark.edu/departments/sociology/research-centers/ communityfamily-institute/resources/communityand-familyinstitute/revised-assessingfood-insecurity-brief.pdf. Accessed 7 Dec 2020; 2020.

8. Wolfson JA, Leung CW. Food insecurity and COVID-19: disparities in early effects for US adults. Nutrients. 2020;12(6):1648.

9. Gundersen C, Ziliak JP. Food insecurity and health outcomes. Health Aff (Millwood). 2015;34(11):1830-9.

10. Lee JS, Gundersen C, Cook J, Laraia B, Johnson MA. Food insecurity and health across the lifespan. Adv Nutr. 2012;3(5):744-5.

11. Holben DH, Marshall MB. Position of the academy of nutrition and dietetics: food insecurity in the US. J Acad Nutr Diet. 2017;117(12):1991-2002.

12. Ke J, Ford-Jones EL. Food insecurity and hunger: a review of the effects on children's health and behaviour. Paediatr Child Health. 2015;20(2):89-91.

13. Pourmotabbed A, Moradi S, Babaei A, et al. Food insecurity and mental health: a systematic review and meta-analysis. Public Health Nutr. 2020;23(10):1778-90.

14. Seligman HK, Berkowitz SA. Aligning programs and policies to support food security and public health goals in the US. Annu Rev Public Health. 2019;40:319-37.

15. Liu Y, Tooze JA, Zhang Y, et al. Breakfast consumption is positively associated with usual nutrient intakes among food pantry clients living in rural communities. J Nutr. 2019;150(3):546-53.

16. Cowan AE, Jun S, Tooze JA, et al. Total usual micronutrient intakes compared to the dietary reference intakes among US adults by food security status. Nutrients. 2019;12(1):38.

17. Rivera RL, Zhang Y, Wang Q, et al. Diet quality and associations with food security among women eligible for Indiana Supplemental Nutrition Assistance Program-Education. J Nutr. 2020;150(8): 2191-8.

18. Wright BN, Tooze JA, Bailey RL, et al. Dietary quality and usual intake of underconsumed nutrients and related food groups differ by food security status for rural, Midwestern food pantry clients. J Acad Nutr Diet. 2020;120(9):1457-68.

19. Weinfield NS, Mills G, Borger C, et al. Hunger in America 2014 national report. Chicago: Feeding America. http://help. feedingamerica.org/HungerInAmerica/hunger-in-america-2014full-report.pdf. Accessed 16 Oct 2020; 2014.

20. Bickel G, Nord M, Hamilton W. United States Household Food Security Survey Module: three-stage design with screeners. Alexandria: US Department of Agriculture, Economic Research 
Service. https://www.ers.usda.gov/media/8271/hh2012.pdf. Accessed 23 Nov 2020; 2006.

21. Laraia BA. Food insecurity and chronic disease. Adv Nutr. 2013;4(2):203-12.

22. Seligman HK, Schillinger D. Hunger and socioeconomic disparities in chronic disease. N Engl J Med. 2010;363(1):6-9.

23. Micha R, Peñalvo JL, Cudhea F, Imamura F, Rehm CD, Mozaffarian D. Association between dietary factors and mortality from heart disease, stroke, and type 2 diabetes in the US. JAMA. 2017;317(9):912-24.

24. Eicher-Miller HA. A review of the food security, diet and health outcomes of food pantry clients and the potential for their improvement through food pantry interventions in the US. Physiol Behav. 2020;220:112871.

25. Leung CW, Epel ES, Ritchie LD, Crawford PB, Laraia BA. Food insecurity is inversely associated with diet quality of lower-income adults. J Acad Nutr Diet. 2014;114(12):1943-53.e2.

26. Gundersen C, Ziliak J. The health consequences of senior hunger in the US: evidence from the 1999-2014 NHANES. Alexandria: Feeding America and the National Foundation to End Senior Hunger. https://www.feedingamerica.org/sites/default/files/ research/senior-hunger-research/senior-health-consequences-2014. pdf. Accessed 16 Oct 2020; 2017.

27. Banerjee S, Radak T. Association between food insecurity, cardiorenal syndrome and all-cause mortality among low-income adults. Nutr Health. 2019;25(4):245-52.

28. Fanelli Kuczmarski M, Brewer BC, Rawal R, Pohlig RT, Zonderman AB, Evans MK. Aspects of dietary diversity differ in their association with atherosclerotic cardiovascular risk in a racially diverse US adult population. Nutrients. 2019;11(5):1034-48.

29. Ford ES. Food security and cardiovascular disease risk among adults in the US: findings from the National Health and Nutrition Examination Survey, 2003-2008. Prev Chronic Dis. 2013;10:E202.

30.• Gregory CA, Coleman-Jensen A. Food insecurity, chronic disease, and health among working-age adults. Washington: Economic Research Service, US Department of Agriculture; 2017. https:// www.ers.usda.gov/publications $/$ pub-details $/$ ?pubid $=84466$. Accessed 16 Oct 2020. Findings from this report suggested a strong association between lower food security with higher probability of three cardiovascular disease related outcomes and seven other chronic diseases examined among workingage US adults at or below $200 \%$ of the federal poverty level.

31. Liu Y, Zhang Y, Remley DT, Eicher-Miller HA. Frequency of food pantry use is associated with diet quality among Indiana food pantry clients. J Acad Nutr Diet. 2019;119(10):1703-12.

32. Palakshappa D, Speiser JL, Rosenthal GE, Vitolins MZ. Food insecurity is associated with an increased prevalence of comorbid medical conditions in obese adults: NHANES 2007-2014. J Gen Intern Med. 2019;34(8):1486-93.

33. Redmond ML, Dong F, Goetz J, Jacobson LT, Collins TC. Food insecurity and peripheral arterial disease in older adult populations. J Nutr Health Aging. 2016;20(10):989-95.

34. Saiz AM Jr, Aul AM, Malecki KM, et al. Food insecurity and cardiovascular health: findings from a statewide population health survey in Wisconsin. Prev Med. 2016;93:1-6.

35. Shiue I. People with diabetes, respiratory, liver or mental disorders, higher urinary antimony, bisphenol A, or pesticides had higher food insecurity: USA NHANES, 2005-2006. Environ Sci Pollut Res Int. 2016;23(1):198-205.

36. Smith MD, Coleman-Jensen A. Food insecurity, acculturation and diagnosis of CHD and related health outcomes among immigrant adults in the USA. Public Health Nutr. 2020;23(3):416-31.

37. Vaccaro JA, Huffman FG. Sex and race/ethnic disparities in food security and chronic diseases in US older adults. Gerontol Geriatr Med. 2017;3:2333721417718344.
38. Venci BJ, Lee SY. Functional limitation and chronic diseases are associated with food insecurity among US adults. Ann Epidemiol. 2018;28(3):182-8.

39. Vercammen KA, Moran AJ, McClain AC, Thorndike AN, Fulay AP, Rimm EB. Food security and 10-year cardiovascular disease risk among US adults. Am J Prev Med. 2019;56(5):689-97.

40. Wang SY, Eberly LA, Roberto CA, Venkataramani AS, Groeneveld PW, Khatana SAM. Food insecurity and cardiovascular mortality for non-elderly adults in the US from 2011 to 2017: a county-level longitudinal analysis. Circ Cardiovasc Qual Outcomes. 2021;14(1):e007473.

41. National Center for Health Statistics. Heart disease. Atlanta: Center for Disease Control and Prevention. https://www.cdc.gov/nchs/ fastats/heart-disease.htm. Accessed 17 Nov 2020; 2020.

42. Goff DC Jr, Lloyd-Jones DM, Bennett G, et al. 2013 ACC/AHA guideline on the assessment of cardiovascular risk: a report of the American College of Cardiology/American Heart Association Task Force on Practice Guidelines. Circulation. 2014;129(25 Suppl 2): S49-73.

43. Leung CW, Zhou MS. Household food insecurity and the association with cumulative biological risk among lower-income adults: results from the National Health and Nutrition Examination Surveys 2007-2010. Nutrients. 2020;12(5):1517.

44. Olson CM, Bove CF, Miller EO. Growing up poor: long-term implications for eating patterns and body weight. Appetite. 2007;49(1):198-207.

45. Eicher-Miller HA, Zhao Y. Evidence for the age-specific relationship of food insecurity and key dietary outcomes among US children and adolescents. Nutr Res Rev. 2018;31(1):98-113.

46. O'Connor DB, Thayer JF, Vedhara K. Stress and health: a review of psychobiological processes. Annu Rev Psychol. 2021;72:4.1-4.26.

47. McEwen BS. Protective and damaging effects of stress mediators. N Engl J Med. 1998;338(3):171-9.

48. McEwen BS. Redefining neuroendocrinology: epigenetics of brainbody communication over the life course. Front Neuroendocrinol. 2018;49:8-30.

49. Berkowitz SA, Basu S, Meigs JB, Seligman HK. Food insecurity and health care expenditures in the US, 2011-2013. Health Serv Res. 2018;53(3):1600-20.

50. Kushel MB, Gupta R, Gee L, Haas JS. Housing instability and food insecurity as barriers to health care among low-income Americans. J Gen Intern Med. 2006;21(1):71-7.

51. Sullivan AF, Clark S, Pallin DJ, Camargo CA Jr. Food security, health, and medication expenditures of emergency department patients. J Emerg Med. 2010;38(4):524-8.

52. Afulani P, Herman D, Coleman-Jensen A, Harrison GG. Food insecurity and health outcomes among older adults: the role of costrelated medication underuse. J Nutr Gerontol Geriatr. 2015;34(3): 319-42.

53. Berkowitz SA, Seligman HK, Meigs JB, Basu S. Food insecurity, healthcare utilization, and high cost: a longitudinal cohort study. Am J Manag Care. 2018;24(9):399-404.

54. Leddy AM, Weiser SD, Palar K, Seligman H. A conceptual model for understanding the rapid COVID-19-related increase in food insecurity and its impact on health and healthcare. Am J Clin Nutr. 2020;112:1162-9.

55. Wolfson JA, Leung CW. Food insecurity during COVID-19: an acute crisis with long-term health implications. Am J Public Health. 2020;110(12):1763-5.

56. Food and Nutrition Service. A short history of SNAP. Alexandria: US Department of Agriculture. https://www.fns.usda.gov/snap/ short-history-snap. Accessed 27 Oct 2020; 2018.

57. Gundersen C, Kreider B, Pepper JV. Partial identification methods for evaluating food assistance programs: a case study of the causal impact of SNAP on food insecurity. Am J Agric Econ. 2017;99(4): 875-93. 
58. Swann CAJFP. Household history, SNAP participation, and food insecurity. Food Policy. 2017;73:1-9.

59. Carlson S. More adequate SNAP benefits would help millions of participants better afford food. Washington: Center on Budget and Policy Priorities. https://www.cbpp.org/sites/default/files/atoms/ files/7-30-19fa.pdf. Accessed 27 Oct 2020; 2019.

60. Keith-Jennings B, Llobrera J, Dean S. Links of the Supplemental Nutrition Assistance Program with food insecurity, poverty, and health: evidence and potential. Am J Public Health. 2019;109(12): 1636-40.

61. Cunnyngham K. Estimates of state Supplemental Nutrition Assistance Program participation rates in 2017. Alexandria: US Department of Agriculture. https://www.fns.usda.gov/SNAP/ estimates-state-snap-participation-rates-2017. Accessed 16 Oct 2020; 2020

62. Eicher-Miller HA, Rivera RL, Sun H, Zhang Y, Maulding MK, Abbott AR. Supplemental Nutrition Assistance ProgramEducation improves food security independent of food assistance and program characteristics. Nutrients. 2020;12(9):2636.

63. Rivera RL, Maulding MK, Eicher-Miller HA. Effect of Supplemental Nutrition Assistance Program-Education (SNAPEd) on food security and dietary outcomes. Nutr Rev. 2019;77(12):903-21.

64. National Institute of Food and Agriculture. SNAP-Ed budget allocation, FY 1992-2018. Washington, DC: US Department of Agriculture. https://snaped.fns.usda.gov/sites/ default/files/documents/SNAP-Ed_Final_Allocations_for_FY_ 2018.pdf. Accessed 20 Nov 2020

65. Food and Nutrition Service. SNAP data tables. In. Alexandria, VA: US Department of Agriculture. https://www.fns.usda.gov/pd/ supplemental-nutrition-assistance-program-snap. Accessed 20 Nov 2020

66. Dietary Guidelines Advisory Committee. Scientific report of the 2020 Dietary Guidelines Advisory Committee: advisory report to the secretary of Agriculture and the secretary of Health and Human Services. Washington: US Department of Agriculture, Agricultural Research Service. https://www.dietaryguidelines.gov/2020advisory-committee-report. Accessed 17 Nov 2020; 2020.
67. Berkowitz SA, Hulberg AC, Standish S, Reznor G, Atlas SJ. Addressing unmet basic resource needs as part of chronic cardiometabolic disease management. JAMA Intern Med. 2017;177(2): 244-52.

68. Dzau VJ, McClellan MB, McGinnis JM, et al. Vital directions for health and health care: priorities from a National Academy of Medicine initiative. JAMA. 2017;317(14):1461-70.

69. Garg A, Toy S, Tripodis Y, Silverstein M, Freeman E. Addressing social determinants of health at well child care visits: a cluster RCT. Pediatrics. 2015;135(2):e296-304.

70. Gottlieb LM, Hessler D, Long D, et al. Effects of social needs screening and in-person service navigation on child health: a randomized clinical trial. JAMA Pediatr. 2016;170(11):e162521.

71. Food and Nutrition Service. Supplemental nutrition assistance program: requirements for able-bodied adults without dependents. Alexandria: US Department of Agriculture. https://www. federalregister.gov/documents/2019/12/05/2019-26044/ supplemental-nutrition-assistance-program-requirements-for-ablebodied-adults-without-dependents. Accessed 17 Nov 2020; 2019.

72. Lowe ET, Poubelle A, Thomas G, Batko S, Layton J. The US Conference of Mayors' report on hunger and homelessness: a status report on homelessness and hunger in America's cities. Washington: National Alliance to End Homelessness. https:// endhomelessness.atavist.com/mayorsreport2016. Accessed 17 Nov 2020; 2016.

73. Wright BN, MacDermid Wadsworth S, Wellnitz A, Eicher-Miller HA. Reaching rural veterans: a new mechanism to connect rural, low-income US Veterans with resources and improve food security. J Public Health (Oxf). 2019;41(4):714-23.

74. Stluka S, Moore L, Eicher-Miller HA, et al. Voices for food: methodologies for implementing a multi-state community-based intervention in rural, high poverty communities. BMC Public Health. 2018;18(1):1055-63.

Publisher's Note Springer Nature remains neutral with regard to jurisdictional claims in published maps and institutional affiliations. 